\title{
A Bump-Hole Strategy for Increased Stringency of Cell-Specific Metabolic Labeling of RNA
}

\author{
Autores \\ Kim Nguyen, Miles Kubota, Jon del Arco, Chao Feng, Monika Singha, Samantha Beasley, Jasmine \\ Sakr, Sunil P. Gandhi, Matthew Blurton-Jones, Jesus Fernández Lucas, and Robert C. Spitale
}

\begin{abstract}
Profiling RNA expression in a cell-specific manner continues to be a grand challenge in biochemical research. Bioorthogonal nucleosides can be utilized to track RNA expression; however, these methods currently have limitations due to background and incorporation of analogs into undesired cells. Herein, we design and demonstrate that uracil phosphoribosyltransferase can be engineered to match 5-vinyluracil for cell-specific metabolic labeling of RNA with exceptional specificity and stringency.
\end{abstract}

